\title{
The Effect of Intermittent Capillary Blood Sampling During Load Incremented Cycling on Physiological, Psychological and Perceptual Variables
}

\author{
Taylor M*, Deldin A, Shafer A, Wisniewski K, Haile L, Panzak G \\ University of the Sciences, USA \\ *Corresponding author: Monica Taylor, University of the Sciences, 600S 43rd St, Philadelphia, PA 19104, USA

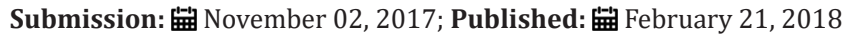

\begin{abstract}
Introduction: The measurement of capillary blood to assess the lactate inflection point is used for health-fitness application and to predict sports performance. However, it is unknown if capillary blood sampling impacts physiological, psychological or perceptual responses during exercise testing.

Purpose: To compare the effect of capillary blood sampling on physiological, psychological, and perceptual during a load incremented cycle protocol.

Methods: A multiple observation, within subject, cross-sectional design was employed. Twenty males (20.4 \pm 2.76 years), and twenty females $\left(22.3 \pm 3.31\right.$ years) performed two load incremented cycle ergo meter tests (Trial A and Trial B) to obtain $\mathrm{VO}_{2}$ peak (lmin $\left.{ }^{-1}\right)$, Ventilation $(V e)\left(1 \mathrm{~min}^{-1}\right)$, $\mathrm{VCO}_{2}\left(\mathrm{lmin}^{-1}\right) . \mathrm{VO}_{2}\left(\mathrm{lmin}^{-1}\right)$, and $\mathrm{HR}\left(\mathrm{bmin}^{-1}\right)$ were recorded each minute. The Vpt was determined as the $\% \mathrm{VO}^{2}$ peak at which Ve: $\mathrm{VO}_{2}$ increased without an accompanying increase in Ve: $\mathrm{VCO}_{2}$. Trial A included capillary blood lactate (BLa) measures taken during the last minute of each stage. Trial B used an identical protocol without BLa measures. The order of administration of Trial A and Trial B were counter-balanced. The psychological/perceptual dependent variables measured during the last 30 seconds of each stage were:
\end{abstract}

1) Affect Valence (AV)

2) Felt-Arousal (FAS)

3) Rating of Perceived Exertion for the overall body (RPE- 0)

4) Exercise Enjoyment (EE) and

5) Perceived intensity level (INT). A paired samples t-test was used to examine between trial differences in psychological and perceptual variables measured at the Vpt along with physiological variables.

Results: There were no significant differences in $\mathrm{VO}_{2}$ peak $^{\prime} \mathrm{HR}_{\mathrm{p}^{\prime}}, \mathrm{TCT}$, and Vpt between Trial A and Trial B ( $\left.\mathrm{p}>0.05\right)$. There were no statistically significant between trial differences found for AV, FAS, RPE- 0 and EE measured at the Vpt. The perceived INT at the Vpt was significantly $\left({ }^{*} \mathrm{p}<0.05\right)$ higher in Trial A than Trial B.

Conclusion: Capillary blood sampling does not appear to influence aerobic fitness variables during exercise testing or most psychological and perceptual variables. This can provide reassurance when incorporating capillary blood sampling into exercise testing protocols.

\section{Introduction}

The measurement of blood lactate to assess and monitor exercise performance is a common practice in sports physiology laboratories worldwide. Lactate analysis is performed for the prescription of training velocities, to evaluate longitudinal changes in aerobic and anaerobic fitness, and to evaluate individual responses to specific training sessions [1]. Most prescriptive research involving blood lactate is directed towards determination of the anaerobic threshold and estimation of the relative anaerobic contribution to exercise based on lactate formation. The theory behind this application is that lactate increases in the blood during heavy to very heavy exercise. As exercise intensity increases above a certain metabolic threshold, anaerobic energy pathways are initiated causing lactate to increase significantly above resting levels. This is accompanied by an almost equal reduction in bicarbonate concentration in the blood causing $\mathrm{CO}_{2}$, production to accelerate, evidenced as an increased respiratory $\mathrm{CO}_{2}$ output. The threshold at which this begins is termed the anaerobic threshold. This threshold has been used as an effective gauge of endurance level in patients with cardio-respiratory disease, healthy normal subjects, and athletes [2]. Some of the common techniques used 
for blood sampling include, finger stick, ear stick and indwelling catheter methods. It has also been observed that blood sampling during exercise may be uncomfortable for subjects being tested; multiple punctures in the same area can be painful, and the site of blood may disturb some participants [3]. Capillary blood sampling may even cause anxiety in some subjects, which could in turn affect test responses. It is currently unknown if capillary blood sampling during exercise testing impacts physiological, psychological or perceptual responses. Therefore the Purpose of this study is to determine the effect of intermittent capillary blood sampling during a load incremented cycle protocol on selected physiological, psychological and perceptual variables.

\section{Methods}

There were 40 total subjects recruited from the university community. They were healthy individuals who participated in aerobic activity 2 days per week for at least 30 minutes each and for no more than 150 total minutes per week. Initially Subjects underwent an orientation session to familiarize them with the incremented cycle protocol and the finger stick method. During the orientation stages (2 minutes in length), capillary blood samples were measured twice, at rest and during the first stage, and subjects were required to cycle to $85 \%$ of there age predicted max heart rate after the Orientation Session subjects participated in 2 experimental sessions; Trial A and Trail B. The order of administration was determined by counterbalance. Each session was separated by 48-72 hours. Before each session Height, Weight and \% Fat were assessed using a Tanita BIA system. The Peak Cycle Ergo-meter Protocol was load incremented and included 3 minutes stages which increased in resistance by 25 watts per stage, women began at 50 watts and men began at 75 watts the subject was required to maintain a continuous $50 \mathrm{rpm}$ pace until exhaustion. The finger stick method utilizing the lactate pro meter was used for all capillary blood sampling procedures.

\section{Measures}

Psychological and perceptual measures were determined by the use of 5 different scales Affect Valance (AV), Felt Arousal (FAS), Ratings of Perceived Exertion (RPE-O), Exercise Enjoyment (EE), and Exercise Intensity (INT). All variables were measured during the last 25 seconds of each minute with the exception of perceived Intensity, which was measured at the end of each stage. AV is defined as the most basic or elementary component of all valenced (positive/negative) subjective responses [4]. Affect valence was measured using a bipolar Feeling Scale [5] ranging from +5 (very good) to -5 (very bad). FAS measures the perceived activation during exercise. High arousal might be experienced as excited, anxious or angry. Low arousal might be experienced as relaxed, bored or calm Arousal was measured using the Felt Arousal scale ranging from 1 (low arousal) to 6 (high arousal). RPE-O is defined as a measure of the intensity of effort, strain, discomfort and/or fatigue that is felt during exercise [6]. Rating of perceived exertion of the overall body was measured using the cycle format 0 (extremely easy) to 10 (extremely hard) Omni RPE scale [7]. EE was defined as "desire to have fun, pursue interests, and/or be stimulated" during exercise participation. The level of exercise enjoyment was measured using a bipolar enjoyment scale that ranged from +5 (very enjoyable) to -5 (very un-enjoyable). INT represents 5 different levels of exercise intensity. The scale ranges from 1 (Light) to 3 (Moderate) to 5 (Vigorous). Moderate exercise intensity was defined as an intensity that noticeably increases your heart rate and breathing. Vigorous exercise intensity was defined as an intensity that substantially increases heart rate and breathing [8-11].

The Ventilatory Breakpoint $\left(\mathrm{V}_{\mathrm{pt}}\right)$ was determined for each subject as the absolute $\mathrm{VO}_{2}$ at which $\mathrm{Ve}: \mathrm{VO}_{2}$ increased without an accompanying rise in Ve: $\mathrm{VCO}_{2}$. The ventilatory breakpoint was expressed as a $\%$ of peak oxygen uptake and served as a dependent variable. Paired-Samples t-tests were used to compare Peak Oxygen Consumption, Heart Rate at $\mathrm{VO}_{2 \text { peak' }}$, Total Cycle Time, and Ventilatory Breakpoint between Trial A and Trial B for males and females [12]. A paired samples t test was also used to examine the differences in psychological and perceptual variables between trials at the ventilatory breakpoint for all psychological and perceptual variables $[13,14]$.

Results

Table 1: Demographics.

\begin{tabular}{|c|c|c|c|}
\hline & Males (n=20) & Females $(\mathbf{n = 2 0})$ & Combined (n=40) \\
\hline Age (yrs) & $20.4 \pm 2.76$ & $22.3 \pm 3.31$ & $21.35 \pm 3.16$ \\
\hline $\begin{array}{c}\text { Height } \\
\text { (cm)* }\end{array}$ & $174.4 \pm 5.82$ & $164.8 \pm 5.40$ & $169.60 \pm 7.37$ \\
\hline Weight (kg)* & $72.08 \pm 8.50$ & $59.98 \pm 5.98$ & $66.03 \pm 9.50$ \\
\hline $\begin{array}{c}\text { Body Fat } \\
\text { (\%)* }\end{array}$ & $13.30 \pm 4.62$ & $23.10 \pm 5.24$ & $18.2 \pm 6.96$ \\
\hline BMI & $23.75 \pm 2.76$ & $22.18 \pm 1.88$ & $22.97 \pm 2.46$ \\
\hline \multicolumn{4}{|c|}{$\mathbf{p = 0 . 0 5}$} \\
\hline
\end{tabular}

Table 2: Mean score of physiological variables at the ventilator breakpoint.

\begin{tabular}{|c|c|c|c|c|c|c|}
\hline & $\begin{array}{c}\text { Comb } \\
\text { A }\end{array}$ & $\begin{array}{c}\text { Comb } \\
\text { B }\end{array}$ & Male A & Male B & $\begin{array}{c}\text { Female } \\
\text { A }\end{array}$ & $\begin{array}{c}\text { Female } \\
\text { B }\end{array}$ \\
\hline VO $_{2}$ peak & 2.81 & 2.8 & 3.23 & 3.29 & 2.38 & 2.31 \\
\hline $\begin{array}{c}\text { Heart } \\
\text { Rate } \\
\text { peak }\end{array}$ & 185.72 & 185.54 & 185.11 & 185.58 & 186.3 & 185.5 \\
\hline $\begin{array}{c}\text { Total } \\
\text { Cycle } \\
\text { Time }\end{array}$ & 15.88 & 15.43 & 17.81 & 16.44 & 13.94 & 14.42 \\
\hline Vpt & $70.68 \%$ & $71 \%$ & $67.20 \%$ & $69.60 \%$ & $74.10 \%$ & $72.40 \%$ \\
\hline \multicolumn{7}{|c|}{$\mathrm{p}=0.05$} \\
\hline
\end{tabular}

Subjects did not differ in age or level of Physical activity (Table 1). Males were significantly taller, weighed more, and were lower in \% body fat when compared to the females [15-17]. There was no significant differences found between Trial A and Trial B for males or females in: $\mathrm{VO}_{\text {2peak }}, \mathrm{HR}_{\text {peak }}$ Total Test Time, or Ventilatory Breakpoint (Table 2). There were also no statistically significant differences between trial A and B for affect, arousal, perceived exertion and exercise enjoyment measured at the $V_{p t}$. The perceived 
intensity at the Vpt was significantly higher in Trial A than Trial B (Table 3). Mean change scores were determined from the end of the first minute for affect, felt arousal, rating of perceived exertion, and exercise enjoyment and the end of the first stage for intensity to immediately post exercise for all variables. No significant differences were found between the mean change scores for any variables.

Table 3: Mean score of psychological variables at the ventilator breakpoint.

\begin{tabular}{|c|c|c|c|}
\hline & Trial A & Trial B & Sig. \\
\hline AV & $1.20 \pm 1.77$ & $1.00 \pm 1.83$ & 0.468 \\
\hline FAS & $3.41 \pm 1.33$ & $3.23 \pm 1.13$ & 0.213 \\
\hline RPE-0 & $5.48 \pm 1.46$ & $5.35 \pm 1.49$ & 0.655 \\
\hline EE & $.95 \pm 1.83$ & $1.00 \pm 1.91$ & 0.824 \\
\hline INT & $2.98 \pm .69$ & $2.63 \pm .84$ & $* .037$ \\
\hline \multicolumn{3}{|c|}{$\mathbf{p = 0 . 0 5}$} \\
\hline
\end{tabular}

In Summary we were able to find that there is no significant differences between Trial A and Trial B for males or females in: $\mathrm{VO}_{\text {2peak }}, \mathrm{HR}_{\text {peak }}$ Total Test Time, or Ventilatory Breakpoint. There were also no significant differences in change score for perceptual and psychological variables. However there was a significant difference in perceived intensity at the ventilatory breakpoint between Trial A and Trial B.

\section{Discussion}

The purpose of this study was to evaluate the impact of capillary blood sampling on physiological $\left(\mathrm{VO}_{2 \text { peak }}, \mathrm{HR}_{\text {peak }}\right.$, Total Test Time, Ventilatory Breakpoint) psychological, and perceptual (AV, FAS, EE, RPE-O, EE, INT) variables during a load incremented cycle protocol to peak. Of particular interest was the behavior of the psychological and perceptual variable in relation to the ventilatory breakpoint. The primary finding of this study was that the inclusion of capillary blood sampling during a load incremented cycle ergometer exercise test does not adversely effect most physiological, psychological or perceptual responses to exercise. However, there was a significant difference in the perception of exercise intensity between Trial A and Trial B. This may have been due to an increased anxiety level of subjects during Trial A as a result of the capillary blood sampling. A Future direction from this study may include the determination of anxiety prior to exercise with the use of an anxiety scale or questionnaire. To determine if anxiety level may have been the cause of the increased perception of intensity. Another direction might be to examine pre test heart rate and blood pressures with an anxiety scale prior to tests. The use of other sampling techniques such as Venipuncture, Ear stick, or the use of an Indwelling catheter could be used along with different exercise protocols such as using a treadmill protocol. Testing specific populations including children, older adults or sedentary individuals could also be a possible direction for further research. Investigators should feel confident proceeding with physiological, psychological and perceptual testing that incorporates blood lactate protocols. In Summary we were able to find that there is no significant differences between Trial A and Trial B for males or females in: V02peak, HRpeak Total Test Time, or Ventilatory Breakpoint. There were also no significant differences in change score for perceptual and psychological variables. However, there was a significant difference in perceived intensity at the ventilatory breakpoint between Trial A and Trial B.

\section{References}

1. Pyne D, Boston T, Martin D, Logan (2000) A Evaluation of Lactate Pro Lactate Analyser. Eur J Appl Physiol 82(1-2): 112-116.

2. Grassi B, Quaresima V, Marconi C, Ferrari M, Cerretelli P (1999) Blood lactate accumulation and muscle deoxygenation during incremental exercise. J Appl Physiol 87: 348-355.

3. Yoshida $\mathrm{T}$ (1984) Effect of exercise duration during incremental exercise on the determination of anaerobic threshold and the onset of blood lactate accumulation. Eur J Appl Physiol 53(3): 196-199.

4. Ekkekakis P, Hall EE, Petruzzello SJ (2004) Practical markers of the transition from aerobic to anaerobic metabolism during exercise: rationale and a case for affect-based exercise prescription. Prev Med 38(2): 149-159.

5. Anderson GS, Rhodes EC (1989) A review of blood lactate and ventilatory methods of detecting transition thresholds. Sports Med 8(1): 43-55.

6. Robertson RJ, Goss FL, Dube J, Rutkowski J, Dupain M, et al. (2004) Validation of the adult OMNI scale of perceived exertion for cycle ergometer exercise. Med Sci Sports 36(1): 102-108.

7. Amann M, Subudhi AW, Walker J, Eisenman P, Shultz B, et al. (2004) An evaluation of the predictive validity and reliability of ventilator threshold. Med Sci Sports 36(10): 1716-1722.

8. Beaver WL, Wasserman K, Whipp BJ (1986) A new method for detecting anaerobic threshold by gas exchange. J Appl Physiol 60(6): 2020-2027.

9. Davis HA, Bassett J, Hughes P, Gass GC (1983) Anaerobic threshold and lactate turn point. Eur J Appl Physiol 50(3): 383-392.

10. Katz A, Sahlin K (1988) Regulation of lactic acid production during exercise. J Appl Physiol 65(2): 509-518.

11. McArdle, WD, Katch FI, Katch VL (2010) Exercise Physiology: nutrition, energy, and human performance. Wolters Kluwer, New York, USA.

12. Pokan R, Hofmann P, Lehmann M, Leitner H, Eber B, et al. (1995) Heart rate deflection related to lactate performance curve and plasma catecholamine response during incremental cycle ergometer exercise. Eur J Appl Physiol 70(2): 175-179.

13. Svedahl K, MacIntosh BR (2003) Anaerobic threshold: the concept and methods of measurement. Can J Appl Physiol 28(2): 299-323.

14. Wasserman K, Beaver WL, Davis JA, PU J, Heber D, et al. (1985) Lactate, pyruvate, and lactate-to-pyruvate ratio during exercise and recovery. J Appl Physiol 59(3): 935-940.

15. Whipp BJ, Koyal SN, Wasserman K (1974) Anaerobic threshold and $0_{2}$ uptake kinetics for work increments of various durations. Med Sci Sports 6: 67-68.

16. Whipp BJ, Davis JA, Torres F, Wasserman K (1981) A test to determine parameters of anaerobic function during exercise. J Appl Physiol 50(1): 217-222. 
Creative Commons Attribution 4.0

International License

For possible submissions Click Here

\section{Your subsequent submission with Crimson Publishers} will attain the below benefits

- High-level peer review and editorial services

- Freely accessible online immediately upon publication

- Authors retain the copyright to their work

- Licensing it under a Creative Commons license

- Visibility through different online platforms

- Global attainment for your research

- Article availability in different formats (Pdf, E-pub, Full Text)

- Endless customer service

- Reasonable Membership services

- Reprints availability upon request

- One step article tracking system 